\title{
The continued use of placebo arms in Covid-19 vaccine trials does not adequately protect the well-being of participants
}

\author{
BRIDGET HAIRE
}

\begin{abstract}
Covid-19 vaccines are a critical tool for controlling the pandemic. While safe and effective vaccines have been developed, research is expected to continue for many years regarding the optimal implementation of existing vaccines in specific settings, and the development of second-generation vaccines that may offer advantages in terms of either efficacy or ease of implementation. Given this context, some commentators have argued that new Covid vaccine trials should be able to use placebo controls, and that existing studies should be able to continue with blinded participants in order to collect high quality, unbiased data. Using international ethics guidance documents, this paper argues against placebo controls, given the existence of proven effective interventions, and against protracted blinding once safety and efficacy milestones have been met. Instead, it advocates for study designs that allow for direct comparison between approved and experimental vaccines, which facilitates both data collection and greater access to vaccines.
\end{abstract}

Key words: Covid-19 vaccines; ethics; placebo controls; equipoise; standards of prevention

At the time of writing, it has been little over a year since the discovery of a novel coronavirus being transmitted between people and causing a spectrum of disease from asymptomatic infection to severe illness and death. Nearly 2.5 million deaths have been reported to the World Health Organisation (1), and the global pandemic has caused massive global disruption, with the necessary implementation of border closures, lockdowns and quarantine on a scale hitherto unseen.

Throughout this period, the development of a safe and

Author: Bridget Haire (b.haire@unsw.edu.au), Kirby Institute, Level 6 Wallace Wurth Building, University of New South Wales, NSW 2052, AUSTRALIA

To cite: Haire B. The continued use of placebo arms in Covid-19 vaccine trials does not adequately protect the well-being of participants. Indian $J$ Med Ethics. 2021 Apr-Jun; 6(2) NS: 122-127. DOI: 10.20529/IJME.2021.20.

Published online first on March 20, 2021.

Manuscript Editors: Ruth Macklin, Amar Jesani.

Peer reviewer: Veena Johari

(c) Indian Journal of Medical Ethics 2021 effective vaccine has been recognised as the only way to start restoring normality. Fast, high coverage roll out on a global scale is the most effective way not only to reduce Covid-19related morbidity and mortality, but also to prevent the development of new variants of SARS-CoV-2, which could potentially be more infectious (as has already occurred with the new strains first found in the UK and South Africa) (2), and/ or more pathogenic. Less virus circulating means fewer opportunities for mutation (3).

Vaccine development has progressed with unprecedented speed (4). There are now four vaccines approved for emergency use by the United States Food and Drug Administration (FDA) (5), with peer reviewed data published on three of these (6-8). A further six vaccines have conditional approval and there are 20 vaccine candidates in large scale efficacy trials, and more than 100 in earlier stages of development (5). Thus, the vaccine pipeline is in great shape, with many potential candidates in development, some of which will soon enter clinical trials.

\section{Vaccine nationalism}

Vaccine roll out is currently highly inequitable. Although the WHO COVAX programme was intended to ensure equity of access globally at least with respect to population size, vaccine nationalism has seen individual high income countries secure contracts with individual vaccine manufacturers, enabling them to stockpile vaccines for their citizens (9). Not surprisingly, this has resulted in inequitable access (10). As Fontanet et al explain, this inequity will drive viral evolution, as dramatic reductions of infection in high income countries only, with infections continuing in low income countries, will result in the emergence of further variants that may not be susceptible to vaccines (3). One of the ways to address this is to deploy greater vaccine coverage in high incidence populations, and to conduct vaccine implementation studies, studies that compare proven effective vaccines head-to-head and studies that compare proven vaccines with new vaccine candidates. In addition to the risk of viral evolution that can occur in settings with high infection rates and suboptimal access to vaccines, there is a risk that countries with poor vaccine access could be deemed guinea pigs by rich countries. As has occurred with perinatal HIV trials, arguments could be made to test new Covid-19 vaccines against placebo, using the 
argument that if populations did not have access to proven effective vaccines, they would not be denied anything if they participated in a trial that used placebo. I will address this "double standard" argument later in the paper.

\section{What are the current research questions about Covid-19 vaccines, and how should they be addressed?}

As Covid vaccines are rolled out in some settings and remain experimental and/or inaccessible in others, important questions are emerging on an almost daily basis. These include the issue of whether some vaccines are effective against emergent variants, (the AstraZeneca efficacy drops below $50 \%$ with some variants)(11) the problem of significantly reduced efficacy in people with HIV (Novavax efficacy dropped from $60 \%$ to $49 \%$ when HIV positive people were included in the analysis)(12) and concerns over deaths in elderly people who had been vaccinated (Pfizer, now shown not to be vaccine related) $(13,14)$. These emerging issues, coupled with important logistic concerns such as the number of doses required for efficacy and cold-chain considerations, point to the major research questions that need to be answered: Which vaccine is best, in a given population or setting? How do the current crop of vaccines stack up against each other, and how do the new candidates in Phase 3 efficacy studies compare? What is the comparative duration of effect? How do they compare with preventing infectivity? Are there rare but clinically significant side effects that will emerge postmarketing?

A recent paper by the WHO Ad Hoc Expert Group for the Next Steps for Covid -19 Vaccine Evaluation (henceforth 'WHO Ad Hoc Expert Group') has argued that (i) the range of research questions that remain with regard to Covid-19 vaccines means that there is still a role for placebo-controlled trials, despite there being proven effective vaccines. In addition, they argued that (ii) it is reasonable to continue trials with blinded participants after a trial has reached its efficacy endpoints in order to continue to collect unbiased blinded data. Regarding placebo-controlled trials, the group argued that running very large but relatively short trials where the active vaccine is tested against placebo is the best way to get clear extended safety data that would capture any very rare but serious safety issues, and prevent adverse events being wrongly ascribed to the vaccine. With respect to keeping participants in Phase 3 trials blinded for periods of follow up after an efficacy results, they argued that this would better facilitate unbiased data on the duration of vaccine effect (15).

In this paper I will make a case against these arguments and will propose that there are strong ethical arguments both at the public health and the individual levels that support a participant-centred model of research, and that the use of placebo controls and delaying participant access to proven effective interventions is antithetical to the equitable distribution of the benefits and burdens of research (16).

\section{Placebo controls in Phase-3 efficacy trials}

The use of placebo controls is generally uncontroversial in instances where there is no proven effective intervention against which to measure the experimental intervention, (16) so the current generation of Covid vaccines have all been tested against placebo. A rationale for placebos being acceptable where there is no proven effective intervention is the principle of "clinical equipoise", defined as genuine uncertainly in the community of experts as to whether an intervention is better than another - or, in the case of a placebo-controlled trial, whether the experimental intervention is better than placebo (17). There have been critiques of this concept (18-20), but Halpern's useful 2006 reformulation of the principle replaces the consensus-based "community of experts" with a clearer, evidence based standard:

Equipoise exists if well-designed studies have yet to answer the question as to which of the two interventions are to be preferred for a particular population of patients (21).

Rules about use of placebos aim to ensure that participants in research studies are not unreasonably denied access to proven effective interventions. In addition to equipoise, the principle of beneficence supports this - that researchers and sponsors should safeguard the health of participants when it is in their power to do so (16). This is addressed in international research ethics guidance documents produced by the Council of Organisations for the Medical Sciences (CIOMS) (16) and the World Medical Association's Declaration of Helsinki (22). The rationale for this is that there is an obligation in a clinical trial to protect the health and wellbeing of study participants, and to ensure that the risks that participants undertake by being in the trial are balanced with benefits (16).

\section{History of the perinatal HIV trials}

It is not a new concept to continue to use placebo after an established effective intervention has been established, even though this violates the principle of equipoise and fails to meet beneficence obligations. In the late 1990s, use of placebo-ased controls despite the existence of proven effective interventions caused a major bioethical controversy (23). Investigators were using placebo controls in low income countries to test interventions to prevent perinatal HIV transmission, although an effective intervention for this had been published in 1994 (24). An intense, polarised debate ensued: some argued that the placebo controls were ethical because the proven intervention was too complex and expensive in low income settings (25), while others argued that this was a double standard, and that research participants in low income countries should be assured the same protections and benefits as those in high income countries (26). 


\section{Positions adopted in the international ethics guidance}

The ethical debate over the use of placebo-controlled trials when proven effective intervention exist, but are not readily accessible was never fully resolved (27). Subsequent revisions of the Declaration of Helsinki and CIOMS take a middle line on this - in general, they advise that an established effective intervention should be used when there is one, but they allow for some exceptions $(28,29)$. CIOMS states:

In sum, when an established effective intervention exists, it may be withheld or substituted with an inferior intervention only if there are compelling scientific reasons for doing so; the risks of withholding the established intervention or substituting it with an inferior one will result in no more than a minor increase above minimal risk to participants; and the risks to participants are minimized (16).

Similarly, the Declaration of Helsinki allows use of a placebo or no intervention only in the following circumstances:

Where for compelling and scientifically sound methodological reasons the use of any intervention less effective than the best proven one, the use of placebo, or no intervention is necessary to determine the efficacy or safety of an intervention and the patients who receive any intervention less effective than the best proven one, placebo, or no intervention will not be subject to additional risks of serious or irreversible harm as a result of not receiving the best proven intervention.

Extreme care must be taken to avoid abuse of this option (22).

As can be seen, both sets of guidance require a compelling scientific justification, and minimal risk to participants to justify an exception. (Of note the "best proven" standard required by the Declaration of Helsinki is a higher standard than the "established effective intervention" required by CIOMS, but this nuance is beyond the scope of the current paper.) I will now consider whether the use of placebos in Covid-19 vaccine trials meets these articulated standards for exceptions.

\section{Is there compelling justification for ongoing use of placebos?}

During this global public health emergency, research into the prevention and treatment of Covid-19 related illness has irrefutably high social value, but are there compelling scientific reasons that support ongoing placebo-based trials? As noted earlier, critical scientific questions currently include which vaccine is best in particular populations and/or particular settings, and whether advantages such as less stringent coldchain requirements can mean that a vaccine with a lower efficacy level can have higher real-world effectiveness, due to easier roll out. Which vaccine works best, if only one dose is administered rather than the full regimen? How do new generation vaccines candidate compare with the first generation of proven vaccines? These types of questions are best answered by comparative trials, such as non-inferiority designs and implementation studies (30). Even the issues of extended safety and duration of vaccine effect raised by the WHO Ad Hoc Expert Group - which they suggested should remain blinded, so that placebo recipients would not be offered the proven effective vaccine for the duration of this study extension - can be answered either observationally or by studies designed to look at proven effective vaccines in comparison with each other, rather than placebo. Thus we can conclude that there is no compelling scientific argument.

Secondly, does a placebo comparator rather than another effective established vaccine comparator, offer no addition risk of serious or irreversible harm? Let us imagine a new Phase 3 efficacy study of a candidate Covid-19 vaccine. If this hypothetical trial were to be held in a population of young, healthy adults who had no risk factors for serious Covid-19 disease, it could be argued that using a placebo control posed only a minimal risk to participants. But would it make sense to limit a Phase 3 trial to such a population? There are already criticisms of the Oxford vaccine, one of the first generation of vaccines, for failing to include sufficient numbers of vulnerable people in trial populations (31). Given the urgent need to evaluate vaccines in people who have risk factors for serious disease, such as pre-existing conditions and/or older age, it is hard to see the scientific rationale for such a trial population. If a trial population were to include people who most need a vaccine - those over 70, and those with comorbidities - then the risk of withholding an established effective vaccine in the comparator arm involves serious and potentially lifethreatening risk.

While the above analysis suggests that Covid-19 vaccine trials do not meet the specified exceptions outlined in the international guidance, an argument to support use of placebos could be made using the precedent from the placebo-controlled perinatal HIV trials: that although Covid-19 vaccines have been proven effective and are being rolled out in many countries globally, they are not yet available in all settings, and thus are not universally established as "standards of care". The premise of this argument is that standards are context-specific, and that participants cannot be said to be harmed if an intervention that they would not otherwise have access to is withheld from them. Returning to the international guidance documents, it is noteworthy that the phrase "standard of care" is not used: the Declaration of Helsinki which has been revised three times since 1996 with particular attention to this clause - uses "best proven"(22) and CIOMS uses "established effective intervention"(16). Neither document makes reference to local standards, where "local standards" can mean no effective intervention, as seen in the perinatal trials. Further, if placebo controls are deemed permissible in settings where there is no current vaccine access outside trials, but impermissible in settings where there is roll out, this would further exacerbate global health inequity given the pre-existing inequities regarding vaccine access between high- and low-income countries. 


\section{Keeping participants blinded after meeting safety and efficacy milestones}

In addition to defending the ongoing use of placebo controls, the WHO Ad Hoc Expert Group also argued that there was a scientific case for continuing trials with participant blinding after prespecified efficacy and safety milestones have been met. The group contended that swiftly unblinding participants and providing active vaccine to placebo recipients would jeopardise the collection of important, unbiased data (15).

This opportunity to obtain reliable evidence about longerterm effects would be destroyed by early unblinding and immediate vaccination of participants assigned to placebo. Although each participant has the option to pursue any available intervention, if substantial numbers of participants choose not to do so, continuation of blinded follow-up in a population in which no licensed vaccine is being deployed could yield important and unexpected findings that would be difficult to obtain reliably any other way (15).

This position contrasts starkly with the perspectives of Covid vaccine trial participants, a group of whom published a letter sent to the FDA about policies regarding unblinding. Firstly, the participant group asked that trial participants who would be eligible to receive a vaccine under the Emergency Use Authorisation be unblinded, so that they may choose to be vaccinated should they be currently on a placebo arm (this addresses the "standards" issue - for those American vaccine participants who now meet criteria for vaccine access, such access may be considered a standard, even though it is an emergency use authorisation).

Secondly, the participants asked that when vaccines in efficacy trials reached their safety and efficacy endpoints, that participants be unblinded and for placebo recipients to have access to the proven effective vaccine facilitated (32). The participants supported their position with ethical and trustbased arguments. Their key ethical argument was reciprocity that as trial participants voluntarily accepted the risk of clinical trial participation, that they should be rewarded when such research produces an effective vaccine. They also argued that delaying access to effective vaccines for trial participants - and particularly those at high risk of severe Covid disease - would undermine trust in the FDA and, by inference, in clinical research (33).

\section{Considering the claims from the Participants' Open Letter}

In evaluating the claims of the participants, it must be recognised that the risk involved in clinical research participation is real - for example, in the HIV field, a Phase 2 trial of a preventative vaccine was halted in 2007 because the vaccine candidate increased the risk of HIV infection (33). Two other trials in the HIV prevention field (vaginal microbicides) also increased risks of infection for participants $(34,35)$. While Covid-19 vaccine research and development has proceeded swiftly and smoothly, the risks undertaken by trial participants were nevertheless real, especially as safety issues in the aforementioned HIV prevention studies only emerged in larger-scale trials so unexpected adverse events can and do occur in such trials. It is also important to note that access to proven effective interventions for trial participants is an ethical norm, and is supported by international research ethics guidance $(16,22,36)$.

Of the three published Phase-3 efficacy studies, the authors of the Pfizer study specifically address the issue of the obligation to research participants.

Although the study was designed to follow participants for safety and efficacy for 2 years after the second dose, given the high vaccine efficacy, ethical and practical barriers prevent following placebo recipients for 2 years without offering active immunization, once the vaccine is approved by regulators and recommended by public health authorities.(6)

This quote demonstrates clearly that it is a realistic, normative expectation that where evidence of efficacy is strong, keeping participants on a placebo arm for a further extended period of time is not fair or reasonable.

While keeping participants blinded following the achievement of safety and efficacy endpoints might enable the collection of further relevant data, such as duration of effect and vaccine impact on infectivity (37), it is both ethically and logistically problematic. To begin with, these data can be collected through ongoing follow-up of all trial participants, after switching placebo recipients to active vaccine - a good precedent for this is the HIV prevention trial that evaluated the impact of early antiretroviral treatment to prevent transmission to sexual partners, which continued collecting data for several years after efficacy was established (38).

The trial participants' letter directly addresses the fact that some of the participants (who are US-based) would now be eligible to receive an emergency use vaccination as priority populations, but that they remain blinded as to whether they have received active vaccine or placebo (32). While as stated earlier there are scientific arguments for continuing without unblinding, such as being able to get high quality data on the duration of vaccine effect (15), failing to unblind after meeting conclusive efficacy endpoints is ethically problematic. Firstly, it violates equipoise, because there is no longer uncertainty as to which arm is more efficacious. Secondly, it does not protect the participants' best interests as required by beneficence, as at least some participants (particularly those in priority populations at increased risk of infection and/or serious disease), would be better off being able to access vaccination should they be eligible. Autonomy is also compromised, as refusal to unblind denies participants the ability to make decisions of importance in their own lives with full information. While some would argue that trial participants have autonomously agreed to participate in the study knowing its conditions, this is contestable as new information 
that comes out during a trial, such as evidence of efficacy, can reasonably change the way a participant thinks about the ongoing risks and benefits of trial participation.

One possible solution to this is for vaccine trials to unblind participants if and when they become eligible under national guidelines. This would mean that those who had priority access to a vaccine would be unblinded, and if they were receiving placebo they could access the active vaccine. In the immediate future, with global vaccine coverage only beginning, this seems a reasonable compromise, if we are willing to discount the argument that all trial participants have taken the risks and all deserve to share in the benefits. This could be justified by the argument that while the continuation of blinding after definitive efficacy endpoints violates equipoise, if it is limited to low risk participants it is unlikely to increase risk of serious disease far above minimum.

\section{What about equipoise and placebos?}

One of the problems with justifying ongoing blinding of participants after definitive efficacy endpoints are reached is the risk that similar reasoning could be applied to future placebo-controlled trials. That is, if trial participants cannot access a proven vaccine in their current setting, it may be permissible to recruit them to a placebo controlled trial, even though there are proven effective interventions elsewhere in the world. If we imagine a few years hence, when some wealthy countries have optimal or near-optimal vaccination coverage and some low- and middle-income countries continue with severely suboptimal access, then we can see how this emphasis on local availability could drive the evolution of double standards. Clinical trialists could take advantage of global inequity in vaccine access to conduct trials with placebos in poor countries that would be impermissible in rich countries. This would be a great moral failure, as it would mean that people in poorer countries would end up disproportionately bearing the burdens of research while rich countries reap the benefits.

\section{Conclusion}

Covid-19 has been a pandemic like no other in terms of its global reach and impact, and vaccines offer an opportunity to control it. Harnessing the potential of vaccines requires both effective governance at the global scale to ensure equitable access to proven effective interventions in all settings, and ongoing research to determine how best to use the available vaccines and whether candidates in development offer advantages over the current generation. Public confidence in the safety and efficacy of vaccines is of course critical for ensuring uptake, and public confidence in the research enterprise is critical to ensuring that people are willing to participate in ongoing trials

Having considered the arguments for further placebo-based trials and for maintaining participant blinding beyond the achievement of key safety and efficacy milestones, I have demonstrated that within the context of Covid-19 vaccines, these actions do not meet the standards required by international ethical guidance documents. In addition to being arguably exploitative, the actions of reducing the benefits that flow to research participants is likely to be a serious disincentive to research participation.

To maintain public confidence in research as an ethical enterprise, adherence to international ethical standards must be upheld, and current or potential participants in Covid-19 vaccine trials need to be assured that their best interests will not be ignored. Large scale vaccine studies in which all participants receive proven effective vaccines can also double as access mechanisms in addition to producing important data.

\section{Competing interests and funding support: None.}

\section{References}

1. World Health Organisation.. WHO Coronavirus Disease (COVID-19) Dashboard: Geneva:WHO; 2021,updated 15 February 2021[cited 2021 Mar 5]. Available from: https://covid19.who.int/ .

2. van Oosterhout C, Hall N, Ly H, Tyler KM. COVID-19 evolution during the pandemic - Implications of new SARS-CoV-2 variants on disease control and public health policies. Virulence. 2021;12(1):507-8.

3. Fontanet A, Autran B, Lina B, Kieny MP, Karim SSA, Sridhar D. SARS-CoV2 variants and ending the COVID-19 pandemic. Lancet. 2021 Feb 11. Doi: 10.1016/s0140-6736(21)00370-6.

4. Knoll MD, Wonodi C. Oxford-AstraZeneca COVID-19 vaccine efficacy. The Lancet. 2021;397(10269):72-4.

5. Zimmer C, Corum J, Sui-Lee W. Coronavirus vaccine tracker New York: New York Times; 2021 [updated 11 February 2021. Available from: https://www.nytimes.com/interactive/2020/science/coronavirusvaccine-tracker.html.

6. Polack FP, Thomas SJ, Kitchin N, Absalon J, Gurtman A, Lockhart S, et al. Safety and Efficacy of the BNT162b2 mRNA Covid-19 Vaccine. N Engl J Med. 2020 Dec 31;383(27):2603-15. doi: 10.1056/NEJMoa2034577. Epub 2020 Dec 10.

7. Baden LR, El Sahly HM, Essink B, Kotloff K, Frey S, Novak R, et al. Efficacy and Safety of the mRNA-1273 SARS-CoV-2 Vaccine. N Engl J Med. 2020 Feb 4;384(5):403-16.

8. Voysey M, Clemens SAC, Madhi SA, Weckx LY, Folegatti PM, Aley PK, et al. Safety and efficacy of the ChAdOx1 nCoV-19 vaccine (AZD1222) against SARS-CoV-2: an interim analysis of four randomised controlled trials in Brazil, South Africa, and the UK. Lancet. 2021;397(10269):99111.

9. Herzog LM, Norheim OF, Emanuel EJ, McCoy MS. Covax must go beyond proportional allocation of covid vaccines to ensure fair and equitable access. BMJ. 2021;372:m4853.

10. Ritchie H, Ortiz-Ospina E, Beltekian D, Mathieu E, Hasell J, Macdonald B, et al. Coronavirus (COVID-19) vaccinations 2021. Available from: . https://ourworldindata.org/covid-vaccinations

11. Mahase E. Covid-19: South Africa pauses use of Oxford vaccine after study casts doubt on efficacy against variant. BMJ. 2021 Feb 8;372:n372.

12. Novavax COVID-19 Vaccine Demonstrates $89.3 \%$ Efficacy in UK Phase 3 Trial [press release]. Novavax, 2021 Jan 28.

13. Torjesen I. Covid-19: Doctors in Norway told to assess severely frail patients for vaccination. BMJ. 2021;372:n167.

14. Kresge N. Pfizer Vaccine Safe for Elderly Despite Norway Scare, WHO Says. Bloomberg. updated 2021 Jan 22 [cited 2021 Mar 5]. Available from: https://www.bloomberg.com/news/articles/2021-01-22/pfizervaccine-safe-for-elderly-despite-norway-scare-who-says.

15. WHO Ad Hoc Expert Group on the Next Steps for Covid-19 Vaccine Evaluation. Placebo-Controlled Trials of Covid-19 Vaccines - Why We Still Need Them. N Engl J Med. 2021 Jan 14;384(2):e2. Doi: 10.1056/ NEJMp2033538. Epub 2020 Dec 2.

16. Council for International Organizations of Medical Sciences (CIOMS) International Ethical Guidelines for Health-related Research Involving Humans,. Geneva; CIOMS; 2016.

17. Freedman B. Equipoise and the ethics of clinical research. N Engl J Med. 1987 Jul 16;317(3). 141-5. 
18. Miller F, Brody H. A critique of clinical equipoise. Therapeutic misconception in the ethics of clinical trials. Hastings Cent Rep. 2003 May-Jun;33(3):19-28.

19. Gifford F. So-called "clinical equipoise" and the argument from design. $J$ Med Philos. 2007 Mar-Apr;32(2):135-50.

20. London AJ. Clinical Equipoise: Foundational Requirement or Fundamental Error. In: Steinbeck B, editor. Oxford Handbook of Bioethics: Oxford University Press; 2009.

21. Halpern SD. Evidence-Based Equipoise and Research Responsiveness. Am J Bioeth. 2006 Jul-Aug;6(4):1-4.

22. World Medical Association. Declaration of Helsinki: Ethical principles for medical research involving human subjects. Fortaleza, Brazil: WMA; 2013 Oct.

23. Lurie $P$, Wolfe SM. Unethical trials of interventions to reduce perinatal transmission of the human immunodeficiency virus in developing countries. N Engl J Med. 1997 Sep 18;337(12):853-6.

24. Connor EM, Sperling RS, Gelber R, Kiselev P, Scott G, O'Sullivan MJ, et al. Reduction of Maternal-Infant Transmission of Human Immunodeficiency Virus Type 1 with Zidovudine Treatment. $N$ Engl J Med. . 1994 Nov 3;331(18):1173-80.

25. Varmus H, Satcher D. Ethical Complexities of Conducting Research in Developing Countries. N Engl J Med. 1997 Oct 2;337(14):1003-5.

26. Angell M. The ethics of clinical research in the Third World. N Engl J Med. 1997 Sep 18;337(12):847-9.

27. Macklin RJ. After Helsinki: Unresolved Issues in International Research. Kennedy Inst Ethics J.. 2001 Mar;11(1): 17-36

28. Skierka A-S, Michels KB. Ethical principles and placebo-controlled trials - interpretation and implementation of the Declaration of Helsinki's placebo paragraph in medical research. BMC Med Ethics. 2018;19(1):24

29. Shrestha B, Dunn L. The Declaration of Helsinki on Medical Research involving Human Subjects: A Review of Seventh Revision. J Nepal
Health Res Counc. 2019;17(4).

30. Dunn DT, Copas AJ, Brocklehurst P. Superiority and non-inferiority: two sides of the same coin? Trials. 2018 Sep 17;19(1):499.

31. Boytchev H. Why did a German newspaper insist the Oxford AstraZeneca vaccine was inefficacious for older people-without evidence? BMJ. 2021 Feb 12;372:n414.

32. COVID-19 vaccine trial participants. An open letter from COVID-19 vaccine trial participants. 2020[cited 2021 Mar 18]. Available from: https://docs.google.com/document/d/ 1 vJdmMbtb8XS2NJJowVhfqlwoTUCitB3Fn7jSbw2kTT8/edit

33. Buchbinder SP, Mehrotra DV, Duerr A, Fitzgerald DW, Mogg R, Li D, et al. Efficacy assessment of a cell-mediated immunity HIV-1 vaccine (the Step Study): a double-blind, randomised, placebo-controlled, test-ofconcept trial. Lancet. 2008 Nov 29;372(9653):1881-93.

34. Van Damme L, Ramjee G, Alary M, Vuylsteke B, Chandeying V, Rees $H$, et al. Effectiveness of COL-1492, a nonoxynol-9 vaginal gel, on HIV-1 transmission in female sex workers: a randomised controlled trial. Lancet. 2002 Sep 28;360(9338):971-7.

35 Van Damme L, Govinden R, Mirembe FM, Guédou F, Solomon S, Becker $\mathrm{ML}$, et al. Lack of Effectiveness of Cellulose Sulfate Gel for the Prevention of Vaginal HIV Transmission. N Engl J Med.. 2008 Jul 31;359(5):463-72.

36. Haire B, Jordens C. Mind the gap: An empirical study of post-trial access in HIV biomedical prevention trials. Dev World Bioeth. 2015 Aug 15(2):85-97. doi: 10.1111/dewb.12039. Epub 2013 Nov 8.

37. Friesen P, Caplan AL, Miller JE. COVID-19 vaccine research and the trouble with clinical equipoise. Lancet. 2021;397(10274):576.

38. Cohen MS, Chen YQ, McCauley M, Gamble T, Hosseinipour MC, Kumarasamy N, et al. Antiretroviral Therapy for the Prevention of HIV-1 Transmission. N Engl J Med. 2016 Sep 1;375(9):830-9.

\section{Thank You, Reviewers!}

We are grateful to our reviewers for the dedicated work they put into evaluating and improving submissions. Besides our core group of Editors, we thank the editorial board members and others who reviewed manuscripts during the year 2020-21:

Aamir Jafarey, Aarthy Ramasamy, Aarthi Raveendiran, Aasim Ahmad, Abha Saxena, Abida Sultana, Akhila Vasan, Alastair McLennan, Alex London, Alok Sarin, Anindita Majumdar, Anjali Nair, Arun Bhatt, Arun Kumar Annamalai, Astrid Lobo Gajiwala, Bernard Dickens, Bevin Vijayan, Bijoya Roy, Bushra Shirazi, Catherine Slack, Charles Weijer, Deepa Pandit, Deepa Venkatachalam, Denny John, Dheeraj Kattula, Dhvani Mehta, Dipika Jain, Eldred Tellis, Eric Meslin, Florencia Luna, George Thomas, Hareesh Angothu, Ido Solt, Ignacio Mastroleo, Ishita Goyal, Jagriti Gangopadhyay, Jasodhara Dasgupta, Jayanta Bhattacharya, Jing-Bao Nie, J Devika, Jissa VT, JK Lakshmi, Joe Varghese, Kenneth Zysk, Ketaki Chowkhani, KS Jacob, Lakshmi Lingam, Lars Breimer, Lopa Mehta, Md Abid Geelani, Madhurima Nundy, Malu Mohan, Manickam Ponnaiah, Manjulika Vaz, Mario Vaz, Monica Sakhrani, Monty Khajanchi, Nagendra Dudi-Venkata, Nandini Kumar, Naveen Kumar, Nithya Gogtay, Nobhojit Roy, Omesh Bharti, Padmaja Shaw, Pragati Hebbar, Pragna Rao, Pravin Bolshete, Priya Satalkar, Priyadarshini C, Priya Pais, Radha D'Souza, Radha Holla Bhar, Raj Kumar Mani, Rakhal Gaitonde, Rakesh PS, Raman Kutty, Ramesh Awasthi, Ravi Prasad Varma, Ravi Vaswani, Reike van der Graaf, Richard A Cash, Rubesh Kumar, Ruth Macklin, Saima Iqbal, Sadhana Natu, Samiran Nundy, Sandeep Bawdekar, Santosh Vijayakumar, Satendra Singh, Saumil Dholakia, Saurav Basu, Sayori Ghoshal, Shahista Parveen, Shaibya Saldanha, Shilpa Phadke, Shining Star Lyngdoh, Shireen Jeejeebhoy, Shrinidhi Datar, Siddharth Sarkar, Smitha Deshpande, Sophia Modi, Soumitra Pathare, Subhasri B, Suchitra Dalvie, Sudarshini Subramaniam, Sudarshan Kottai, Sudha Ramalingam, Sujit Chandy, Sundar Sarukkai, Sunil K Pandya, Sunil Rajpal, Sunu C Thomas, Supriya Subramani, Suthanthira Kannan, Swarnalakshmi Singaravelu, Sylvia Karpagam, Upreet Dhaliwal, Usha Raman, Vallish BN, Varalakshmi Elango, Vipin Vashishtha, William Joe, Yogesh Jain, 